\title{
Modeling and Optimization of Deflection Slits for Fast-Pulsing a Low Energy Ion Beam
}

Author: Ryan Bosca

Faculty Mentor: Duncan Weathers, Department of Physics, College of Arts and Sciences

Department: Department of Physics, College of Arts and Sciences

\& Honors College 


\section{Bio:}

Ryan Bosca graduated cum laude and with Distinguished Honors from UNT in May 2006 with a bachelor's degree in physics. He has participated in Research Experiences for Undergraduates, funded by the National Science Foundation during the summers of 2003 and 2004 at UNT. He presented this research at the Texas Section of the American Physical Society in October 2004 at Baylor University. This research was presented at Scholar's Day 2006 and at the National Conference on Undergraduate Research 2006, where it was published in the conference proceedings. He is currently attending graduate school at UNT working toward a Master of Science in Physics. 


\begin{abstract}
:
When a transverse electric field between a pair of closely spaced slits is used to deflect an ion beam, the length of the field region along the beam axis directly affects the length of time required to make the transition between the beam's undeflected and deflected states. Using the SIMION $^{\mathrm{TM}}$ 3D 7.0 ion optics simulation program, which calculates electrostatic fields via numerical solutions to Laplace's equation, we have modeled a set of deflection slits for fastpulsing a keV ion beam. We used the electric fields calculated in the model to simulate ion flight through the deflection region. By establishing a condition for ion transmission to a downstream target and by starting ion flights at different positions along the beam axis in the electric field region, we have obtained the time dependence for the beam's transition from the undeflected to the deflected state on the downstream target, assuming negligible propagation times of the electric field through the deflection region. We compare the simulated deflection to the measured performance of a deflection system on a small Cockcroft-Walton accelerator. We also investigate simulated slit configurations that reduce the length of the fields along the beam axis, thus optimizing the pulsing mechanism.
\end{abstract}




\section{Introduction}

Certain experimental techniques such as time of flight (TOF) measurements, material analysis, and material deposition require the use of a pulsed ion beam. Researchers employ a variety of experimental setups to accomplish ion beam pulsing. Pulsing the ion source is one such method. Bunching is another pulsing technique in which an oscillating voltage accelerates part of the beam, allowing the accelerated ions to catch up to slower moving ions in front, thus creating a pulse of ions. A much different method requires passing ions through closely spaced electrodes with an adjustable voltage between the slits. Using transverse electric fields, created by voltage differences between the slits, allows for deflection of the beam from the optical axis. Conversely, the absence of transverse electric fields or lack of a voltage difference between the slits allows ions to pass through the region undeflected. By rapidly alternating between these two states, it is possible to produce a pulsed ion beam. Exeler, et al. used such a system in which they produced a $1 \mathrm{keV} \mathrm{H}^{+}$pulsed ion beam using a transverse electric field between two electrodes to deflect ions across an aperture. ${ }^{1}$ The system under study at University of North Texas (UNT) utilizes a similar system in which a small Cockcroft-Walton accelerator produces ions upstream from a pair of vertically oriented electrodes, positioned to coincide with the most tightly focused portion of the beam, that produce transverse electric fields between the electrodes to deflect ions into a physical boundary, which ends beam current on target. ${ }^{2}$ The pulsed beam is produced by maintaining one slit at a constant 228 volts (V), which is the present limit of the pulse generator, while voltage on the other slit is rapidly changed between $0 \mathrm{~V}$ and $228 \mathrm{~V}$ and then back to $0 \mathrm{~V}$. This rapid change between voltages removes the transverse electric fields, allowing ions to reach the target, and then reinstates the fields causing deflection of the ions into physical boundaries to 
create the pulse. SIMION ${ }^{\mathrm{TM}}$ 3D 7.0, hereafter referred to as SIMION, ion optics simulation program was used to model this process via numerical calculations of ion trajectories through experimental apparatus at UNT. From this data, a temporal relationship describing the ion beam pulse was constructed. In the application being modeled, the pulsed beam is used to initiate TOF measurements and the temporal width of the ion pulse has been the limiting factor in attainable resolution with TOF measurements. The purpose is to model the present configuration of the apparatus and to explore new configurations that might allow for increased resolution by decreasing the temporal width of the ion pulse.

\section{Methodology}

The experimental configuration simulated in this work is shown schematically in Figure 1. Using SIMION, the analyzing magnet, entrance and exit slits, and beam blocker were modeled. For regions containing either electric or magnetic fields, SIMION calculates numerical solutions to those fields, which it uses to determine ion trajectories through those regions. Once the program calculates the fields, a collection of ions is defined with a suitable distribution of initial positions and directions to represent operation of the ion beam. SIMION then simulates the flight of the ion beam through the modeled setup and it records the TOF of all ions reaching the final target. The recorded data are used to construct a temporal relationship describing the beam current as a function of time as described in more detail next.

\section{Modeled Experimental Setup}

In the experimental apparatus, the beam from the accelerator is focused on the entrance slits to an analyzing magnet. Ions with the appropriate momentum to charge ratio are deflected 
by 45 degrees in the magnet and are transmitted through the beam blocker and then the exit slits downstream from the magnet. Ions transmitted through the exit slits bombard the target, which produces the beam current on the target.

Models for individual optical elements were produced using SIMION. Elements modeled include the entrance slits, magnet, beam blocker, exit slits, and target. Each element is defined in a simulation volume called a potential array, in which points are defined as either electrode points or non-electrode points. Here, the electrodes were defined to match the actual physical dimensions of the experimental apparatus. Potential arrays containing either electric or magnetic fields were refined, a process in which SIMION calculates the fields via numerical solutions to Laplace's equation using an over-relaxation method. ${ }^{3}$ Due to the discrete nature of the potential array, field calculations near electrode surfaces become inaccurate at distances less than 1 grid unit away from electrodes. For this reason, the highest density of grid units possible was used for each potential array to smooth the surfaces of optical elements that interact with ions and thus improve field accuracy.

The pulsing slit's region included the upstream flange face that couples to the remainder of the upstream vacuum housing, the pulsing slit, which is oriented vertically, an adjacent horizontal slit located just downstream that acts as an aperture, and the grounded ion beam line tubing. To model the electric fields as accurately as possible within this region, the electrode density was set to 4 grid units per millimeter. An axis representing the optical axis of the beam line was defined to pass midway between the slit electrodes with its origin in the plane of the flange face upstream from the slits. Using this configuration, an accurate model of the electric fields was calculated in the region of the pulsing slits. However, due to program limitations, it 
was not possible to model portions of the electric fields that extended beyond the upstream flange face while accurately modeling fields close to the pulsing slit; this limitation is discussed later.

After ions exit the region of the pulsing slits, they travel through an aperture in a beam blocker located between the poles of the bending magnet. Both the bending magnet and beam blocker were modeled with an electrode density of 10 grid units per millimeter. With the magnetic fields calculated and the magnet correctly aligned to the optical axis, the field intensity was then calibrated for a $35 \mathrm{keV} \mathrm{Ar}{ }^{+}$ion beam such that the field deflected an ion flying directly along the optical axis by 45 degrees. With this calibration, the beam blocker was then positioned with its aperture centered on the optical axis.

Downstream from the beam blocker are the exit slits, which determine final ion transmission to the target. The transmission condition imposed by these slits is largely dependent on the energy of each ion. Only the vertically oriented downstream slits were modeled in the final experimental setup. Because no voltages are applied to either of the exit slits, they act purely as an aperture, and only the vertically oriented slit is needed. In the simulation, these slits were centered on the optical axis such that the front face of the slit was perpendicular to the axis. Finally, a target was placed at the end of the beam line and centered on the optical axis. This physical barrier was used to terminate ion flight through the simulation and facilitate data recording.

\section{Ion Definition}

An initial beam profile was constructed in the plane of the front face of the pulsing slit, corresponding to the position of a beam waist ${ }^{4}$ in the horizontal direction. The beam was defined 
to have a flat, uniformly distributed profile in phase space, illustrated in Figure 2. SIMION was used to calculate the propagation of this profile both upstream and downstream from the front face of the pulsing slits while the slits were in the undeflecting state (both slit electrodes at a potential of $228 \mathrm{~V}$ ). At set time intervals of 0.6 nanoseconds (ns), corresponding to a spacing of about $0.2 \mathrm{~mm}$ along the optical axis, the program recorded the position, angle, and energy of each ion. The data were recorded to an ASCII file and then imported into a spreadsheet program, where they were converted into an ion definition file. Ions with upstream velocities were reversed to fly in the direction of the ion beam. These files constituted the simulated undeflected ion beam and were used to simulate the turn-off portion of the beam pulse.

To simulate the turn-on portion of the beam pulse, ions were flown upstream from the waist to the beginning of the potential array, with the pulsing slit in the undeflected state. Data were recorded for all ions at that position. A spreadsheet was used to import the data and reverse the direction of flight, and this was recorded into ion definition files. These ions represented the beam from the accelerator. The slit was changed to the deflecting state (one electrode grounded, one electrode at $228 \mathrm{~V}$ ), the ions were then flown, and data were recorded every $0.6 \mathrm{~ns}$ to define the deflected ion beam in the deflection region.

A 100 ns beam pulse was generated by defining ions in the following manner. Ions were defined by flying the ion definitions for the turn-on portion of the pulse $100 \mathrm{~ns}$ and recording ion data while the slit was in the undeflecting state. Then, the first set of data that were recorded for the turn-on ion definitions was flown downstream until the ions were within approximately 0.2 $\mathrm{mm}$ of the previous set of ions that were flown, and data were recorded every 6 ns. In Figure 2, 
each point represents an individual ion. Distance given is the $x$ distance off the optical axis. The angle is measured between an ion's trajectory and the optical axis.

\section{Temporal Relation}

To simulate the turn-off of the ion beam, the electrodes in the simulation were set to the deflecting state, and the ions defined for the undeflected beam described above were flown through the entire modeled optical system. SIMION calculates the total TOF of all ions that impinge ("splat") on a physical boundary in the simulation. Only ions that were transmitted to the final target were included in constructing the temporal relation. These ions were identified using coordinate position data. A spreadsheet program was then used to bin ions that reached the final target into time intervals of $0.6 \mathrm{~ns}$, and a histogram was made, as displayed inabove..

To simulate the turn-on of the ion beam, the same procedure was used, except that the electrodes in the simulation were set to the undeflecting state, and the ions defined for the deflected beam described above were flown through the entire modeled optical system.

By using this procedure, we effectively simulate a dynamic process involving timedependent electrode voltages using a program that only models static fields. Note that this method as described does not account for the finite rise and fall times of the electrode voltage in the deflection system, but rather assumes that it changes instantaneously.

\section{Data}

After running all simulations, the recorded data were exported to a spreadsheet where the temporal relationship was constructed as described earlier. Simulations were run on two different physical configurations of the pulsing slit, which are displayed in Figure 7. The geometry referred to as the "present configuration" reflects the design of the existing deflection system 
hardware, in which thin blades that form the slit opening and their respective clamps are at the same potential. The geometry referred to as the "adjusted present configuration" has the same physical dimensions as the first geometry, but the blades are modeled as being electrically isolated from the clamps, with the clamps grounded. A third configuration, which incorporates a three-slit design, differs significantly from the first two. In this configuration, the front and back slits are grounded, while the center slit is electrically isolated and acts in the same manner as in the other two configurations.

\section{Turn-Off Data}

Using the present configuration, we found that the time required for the ion current at the final target to decay from its maximum value to zero was approximately $80 \mathrm{~ns}$. The simulation results showing this decaying ion current, which corresponds to the turn-off portion of the beam pulse, are presented in Figure 3a. The new configuration exhibited a significantly faster turn-off time of approximately $34 \mathrm{~ns}$, as can be seen in Figure 3b.

\section{Turn-On Data}

Running the simulation with the present configuration yielded a turn-on time of approximately $60 \mathrm{~ns}$, which is illustrated in Figure 4a. Similar to the turn-off data, the turn-on data for the adjusted present configuration resulted in a sharp decrease in time required to transition from the deflected to the undeflected state. Overall, the time required to transition was about $36 \mathrm{~ns}$, as seen in Figure 4b.

\section{0 ns Beam Pulse}

The major limiting factor in the ability to reduce pulse width with the present configuration is that for pulses shorter than about $150 \mathrm{~ns}$, the maximum current pulse amplitude 
on the final target decreases with decreasing pulse width. This can be seen from a simulation of the pulse that drives the variable electrode of the pulsing slit, and is a direct consequence of the slow transition time between the deflected and undeflected states of the ion beam. With the current configuration, we found that the amplitude of a 100 ns pulse was reduced by $15 \%$, from the maximum possible amplitude for longer pulses. The simulated pulse, shown in Figure 5a, reaches a maximum ion count of 374 , compared to a maximum possible ion count of 441 . This simulated result closely matches the measured ion beam pulse, an oscilloscope trace of which is shown in Figure 5b, along with a trace of the generating pulse applied to the variable electrode of the pulsing slit, shown in Figure 5c. We also simulated a 100 ns pulse using the adjusted configuration. This simulation displayed marked improvement in maximum attainable current; we saw a maximum, almost steady-state, at an ion count of 431 ions, as displayed in Figure 6. From the figure, it is apparent that the pulse width for this configuration could be reduced by at least $40 \mathrm{~ns}$ before appreciably affecting the pulse amplitude.

\section{Discussion}

The two simulated setups as discussed here and a third model that will be simulated are displayed in Figure 7 with blue lines that represent contours of constant electric field magnitude. The difference in field magnitude between adjacent lines is 2.5 volts per millimeter; the field decreases with increasing distance from the deflection electrode. These contours indicate the extent of the deflection field region. The fields in the present configuration extend beyond the boundary of the figure (and the simulation), which in turn results in long turn-on and turn-off times. Because of the small magnitude of the extended fields, however, their effect should be negligible in determining the temporal relationship of the beam pulse. Fields in the adjusted 
experimental setup are confined much more closely to the electrode area, which results in the more rapid beam pulse transitions. Electric fields are further confined by the three-slit design, which we anticipate will reduce turn-on and turn-off times even more.

Despite the close correlation of experimental results and data, the inaccuracy of fields close to electrode surfaces needs to be addressed. The current model has a gap spacing between the electrodes of one $\mathrm{mm}$, which corresponds to 4 grid units. Because of the discrete definition of electrodes, ions flying within 1 grid unit of the surface may have inaccurate trajectories. This will be discussed in further work.

\section{Conclusion}

We have simulated the dynamic process of pulsing an ion beam, using the static capabilities of the ion optical simulation program SIMION. In modeling a configuration based on the existing experimental design in our laboratory, we have found that the electric fields responsible for pulsing the ion beam extend well beyond the pulsing slit, resulting in slow transition times from the deflected to the undeflected state and vice versa. The simulated shape of the beam pulse was found to agree well with the experimentally measured beam pulse. A simulation using a geometry that better confined the deflecting field region produced markedly sharper transition times for the ion beam. Finally, a third deflection geometry has been proposed that promises to shorten transition times even further. 


\section{Notes}

1. Exeler, N., Rathmann, D., \& Willerding, B. (1985). "Ion beam pulsing for time of flight experiments" Journal of Physics E: Scientific Instrumentation. 18: 17.

2. Bigelow, A. W., Li, S. L., Matteson, S., \& Weathers, D. L. (1999). "Sputter-Initiated Resonance Ionization Spectroscopy at the University of North Texas," CP475, Applications of Accelerators in Research and Industry. New York, NY: AIP Press.

3. Dahl, D. A. (2000). SIMION 3D Version 7.0 User's Manual, Idaho Falls, ID: Bechtel BWXT.

4. Wollnik, H. (1987).Optics of Charged Particles. San Diego: Academic Press, Inc. 
Figure 1. Experimental schematic.

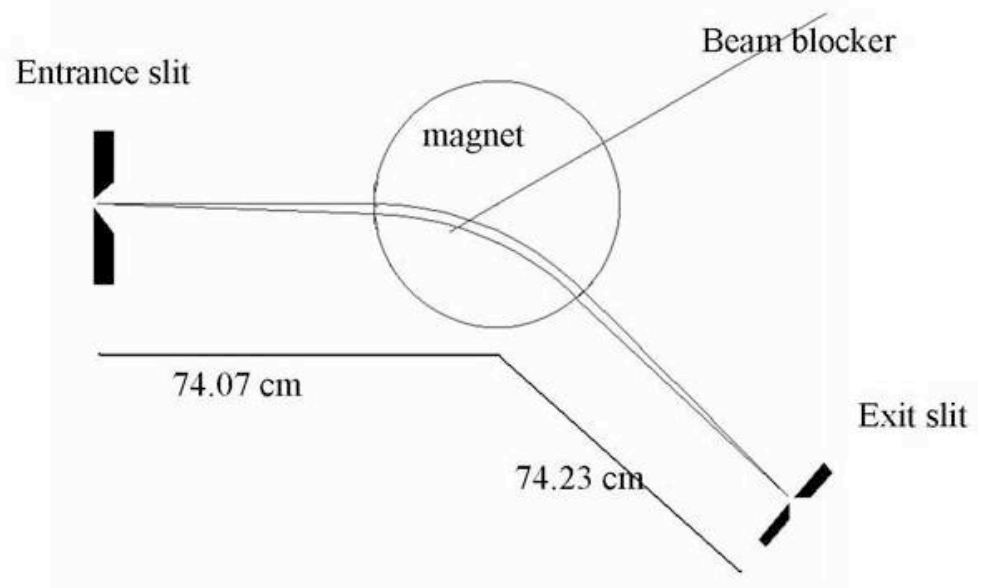


Figure 2.

Phase space of the undeflected ion beam in the plane of the pulsing

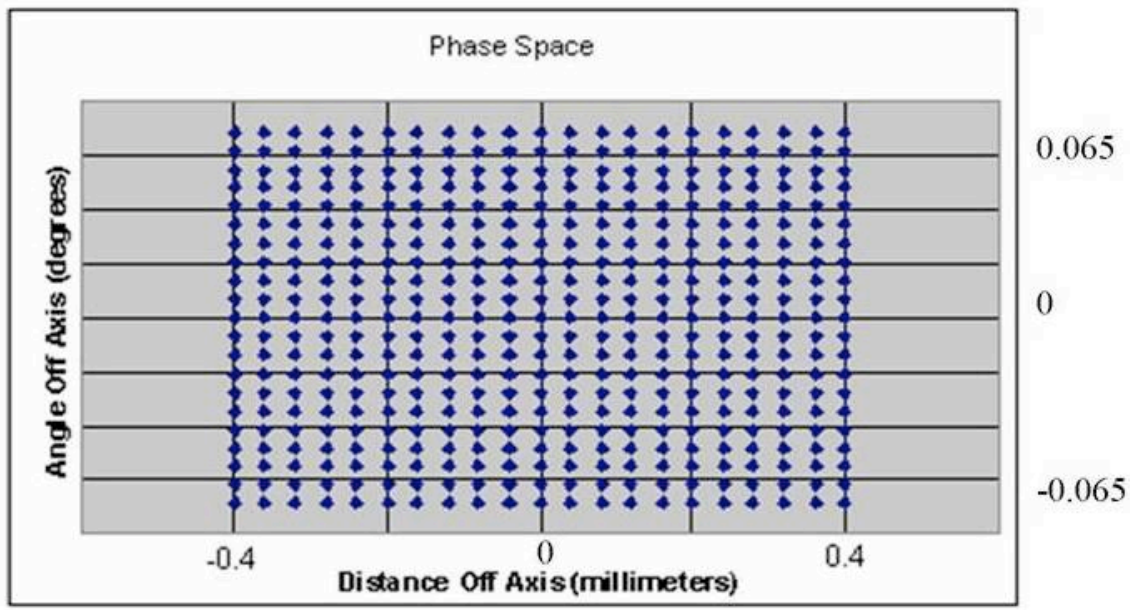


Figure 3a. Turn-off time of current configuration.

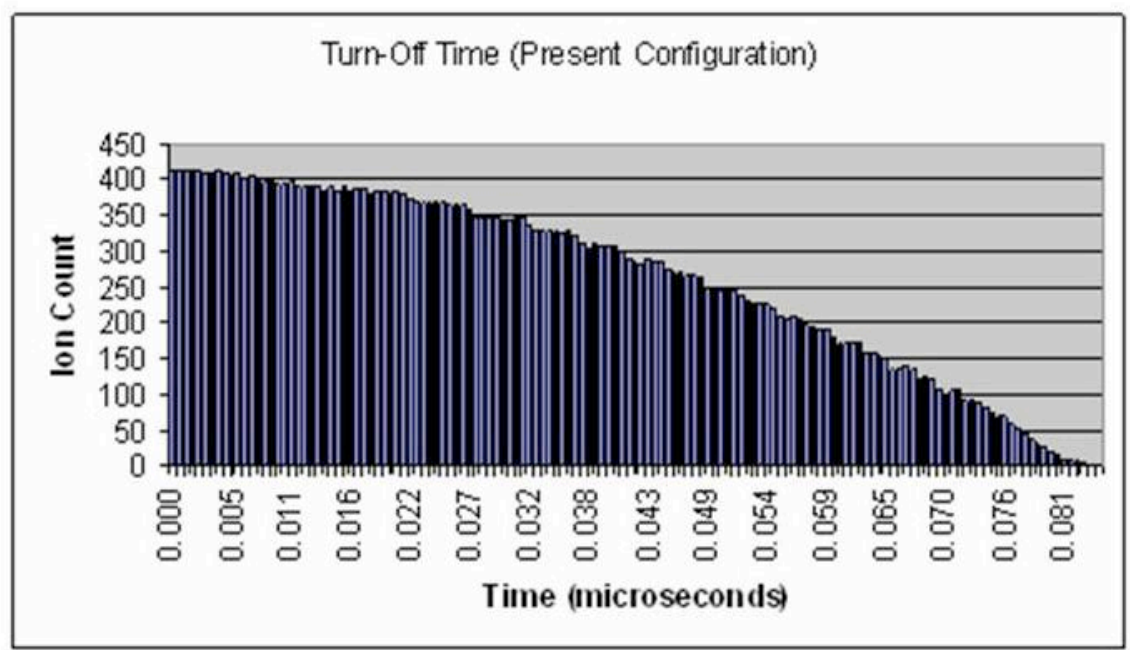


Figure $3 b$. Turn-off time of adjusted current configuration.

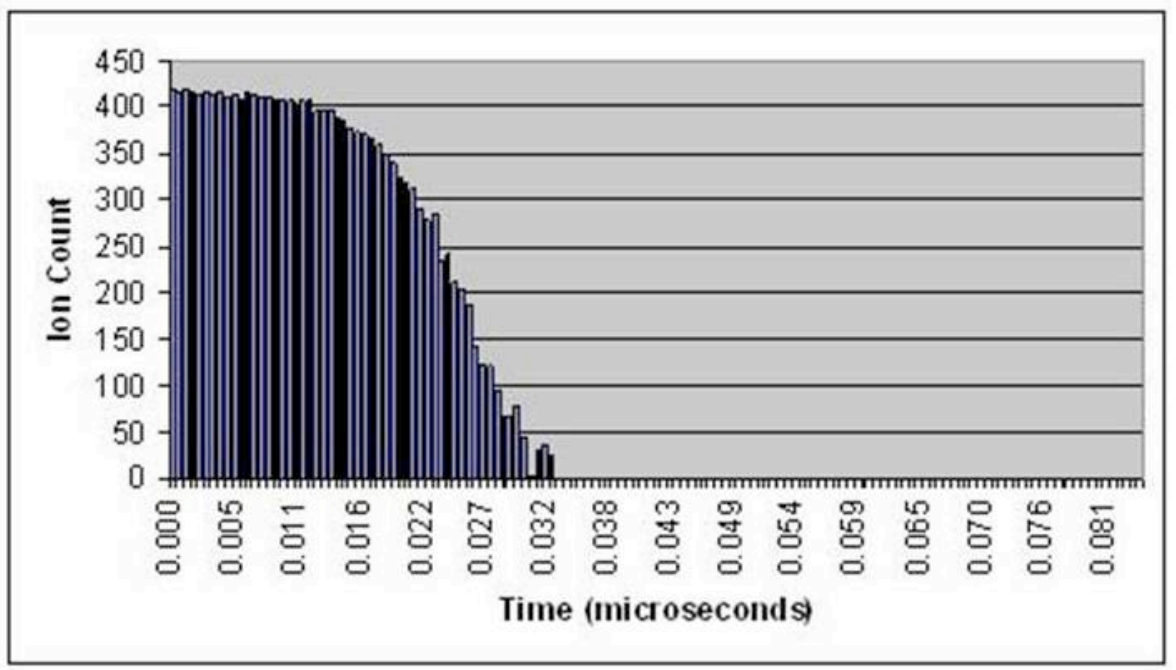


Figure 4a. Turn-on time of current configuration.

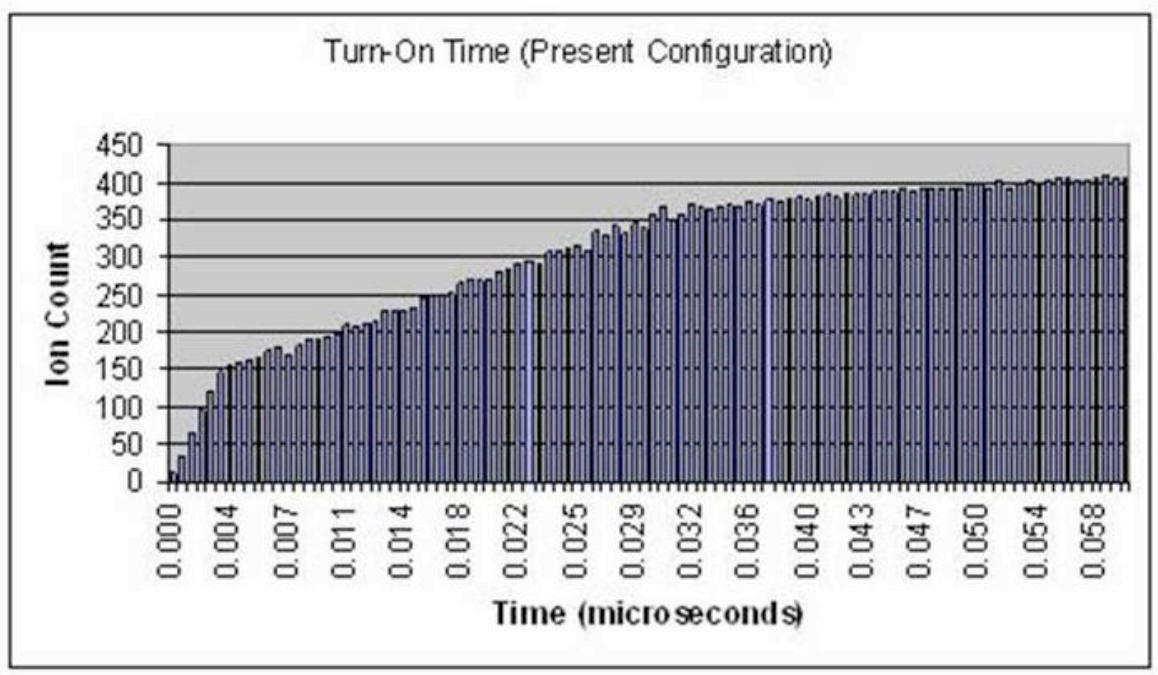


Figure $4 \mathrm{~b}$. Turn-on time of current adjusted configuration.

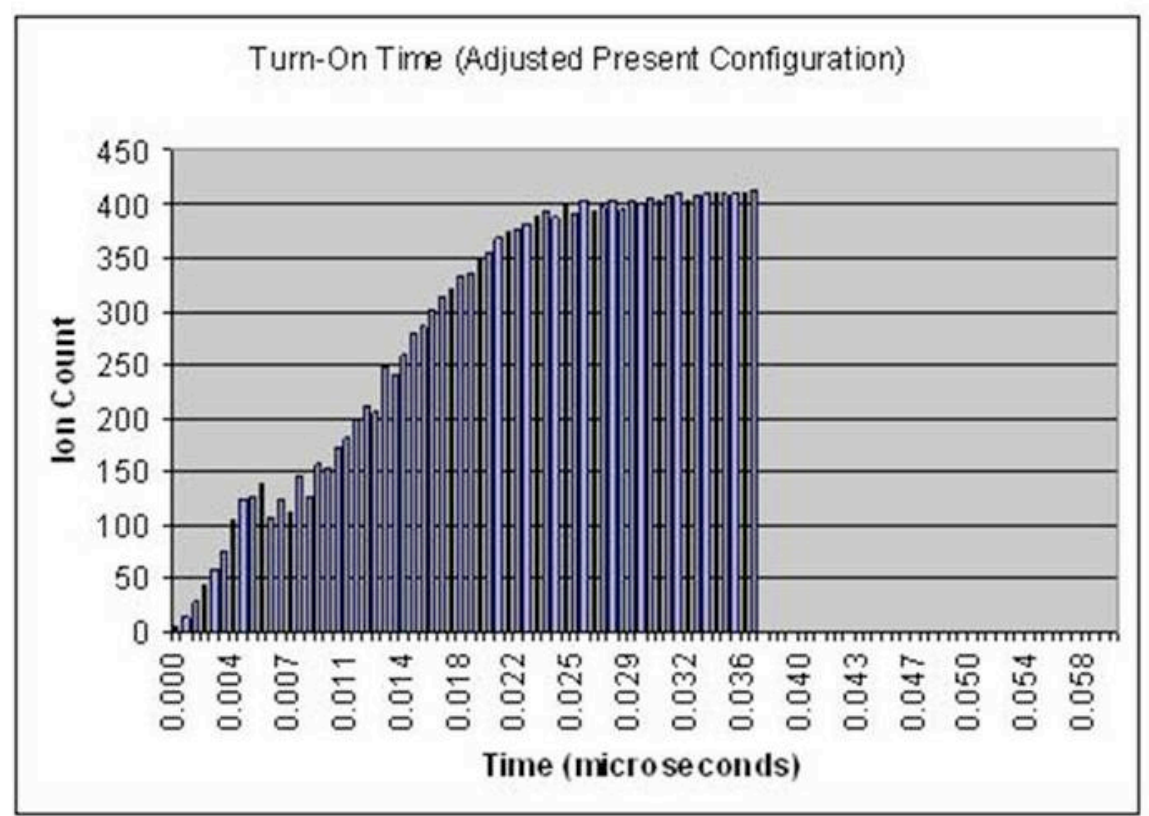


Figure 5a. 100 ns pulse with current configuration.

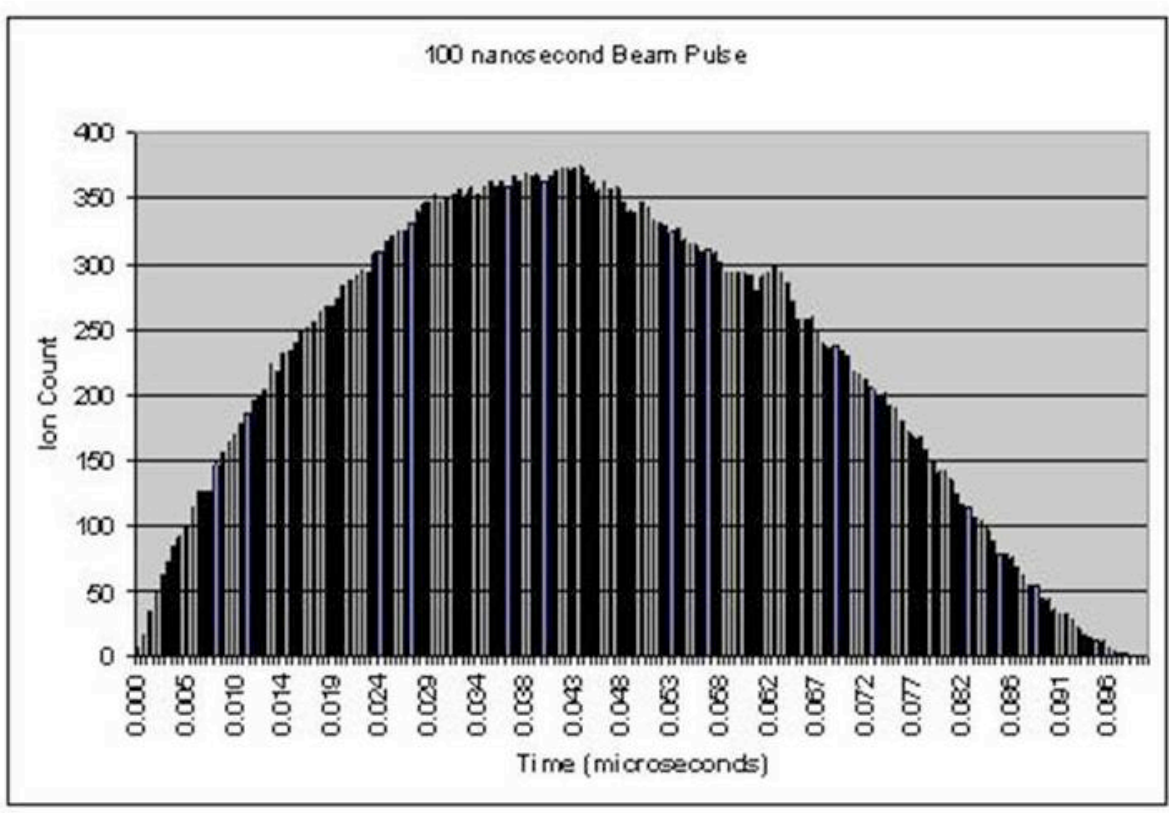


Figure $5 \mathrm{~b}$. Oscilloscope trace of beam pulse (left). Oscilloscope trace of pulse generator on variable electrode (right)
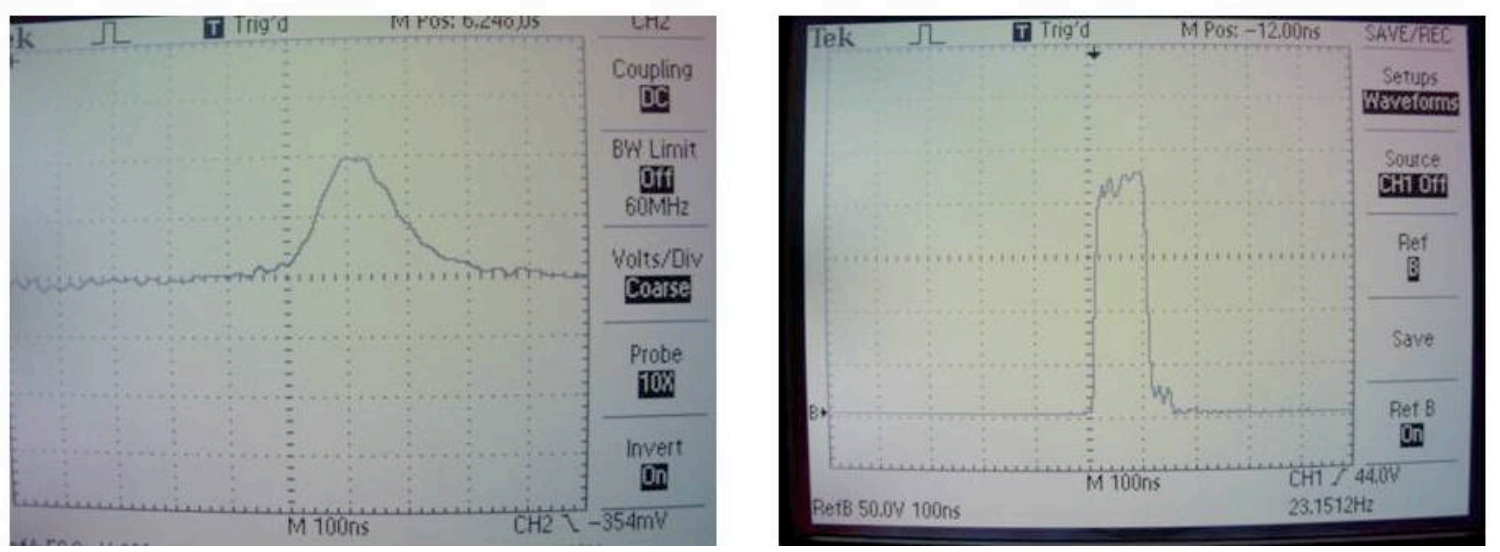
Figure 6.100 ns beam pulse with adjusted current configuration.

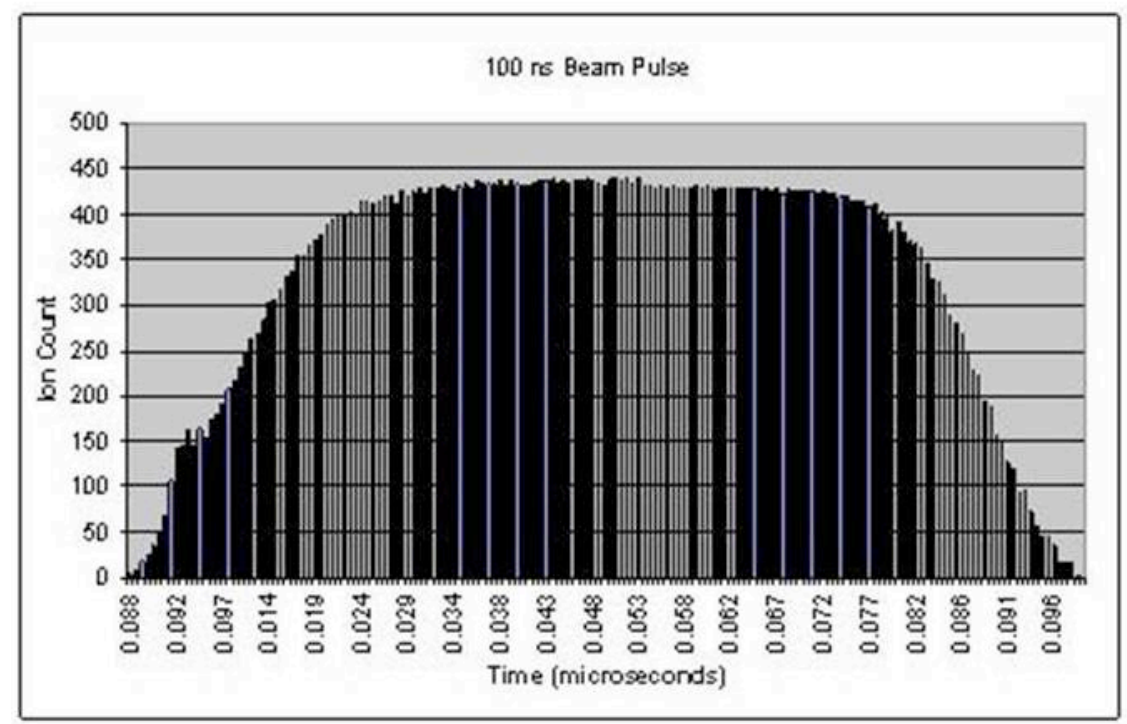


Figure 7. Present configuration (leff), adjusted present configuration (middle), three-slit configuration (right), with electric field contours displayed.
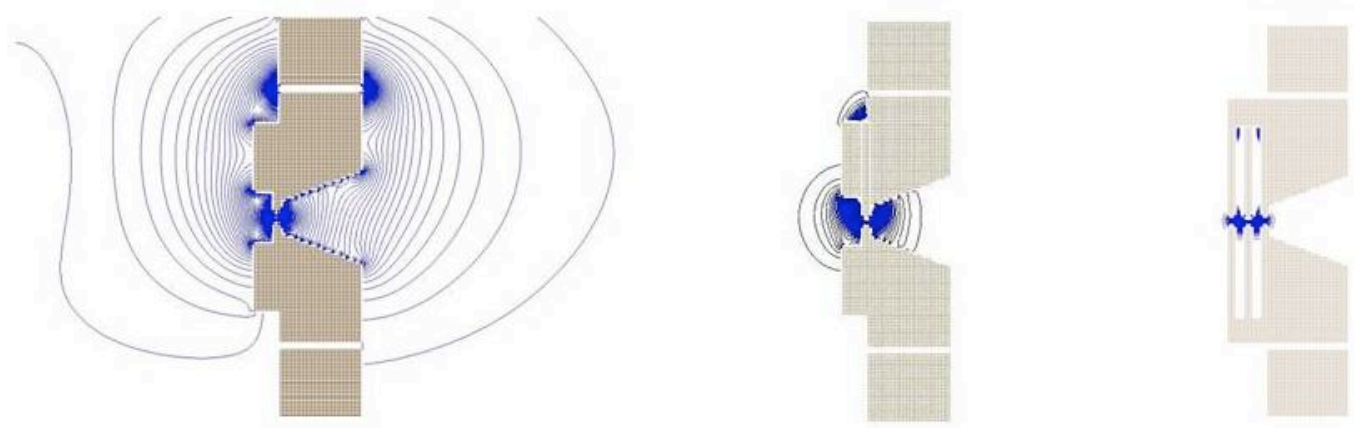